\title{
The D/H Ratio in Interstellar Gas Toward the Hot, White Dwarf G191-B2B
}

\author{
M. S. Sahu \\ NASA/Goddard Space Flight Center, Code 681, Greenbelt, MD 20771 \\ and National Optical Astronomy Observatories, 950 North Cherry \\ Avenue, Tucson, AZ 87519-4933
}

\begin{abstract}
.
Space Telescope Imaging Spectrograph (STIS) observations of the $\mathrm{D} / \mathrm{H}$ ratio in the two velocity components towards G191-B2B are consistent with $1.5 \pm 0.1 \times 10^{-5}$ and do not agree with the values derived using the Goddard High Resolution Spectrograph (GHRS) data. We present some new work on the G191-B2B sightline, and the results we obtain are consistent with those of Sahu et al. (1999).
\end{abstract}

\section{Introduction}

The fraction of deuterium not processed into helium during primordial nucleosynthesis is a sensitive function of the cosmological baryon density $(\eta)$ and can be used to probe conditions in the earliest times of the Universe. Deuterium is destroyed when it is cycled through thermonuclear processes in stars and there is no reasonable way other than $\mathrm{BBN}$ to produce any significant quantity of deuterium in the Universe (Epstein, Lattimer \& Schramm, 1976). Since the amount of deuterium in the Universe decreases monotonically with time, the $\mathrm{D} / \mathrm{H}$ abundance ratio in the Local Interstellar Medium (LISM) places a lower limit on the density of baryons in the Universe.

In the early 1970's, observations of interstellar deuterium toward early-type stars with the Copernicus satellite provided the first strong evidence that the Universe is not closed by baryons and that a significant amount of non-baryonic dark matter should be present (Reeves et al. 1973). More recently, deuterium abundance measurements in metal-poor Lyman- $\alpha$ clouds toward high-redshift QSOs have provided more direct measurements of the primordial deuterium abundance (e.g. Burles \& Tytler, 1998). The more detailed D/H studies possible in the LISM now focus on testing the Galactic chemical evolution models of deuterium, and, in particular, on testing the assumption that there is no significant non-cosmological deuterium production.

Hubble Space Telescope (HST) - Goddard High Resolution Spectrograph (GHRS) measurements of the $\mathrm{D} / \mathrm{H}$ ratio in the Local Interstellar Cloud (LIC), using nearby, late-type stars and WDs as background sources are all consistent with a uniform $\mathrm{D} / \mathrm{H}$ value of $1.5 \pm 0.1 \times 10^{-5}$ (e.g. Linsky et al. 1993, 1995, 
hereafter L93 \& L95; Linsky 1998, Landsman et al. 1996, Dring et al. 1998). This view of the constancy of the $\mathrm{D} / \mathrm{H}$ ratio in the LISM was questioned by the results of Vidal-Madjar et al. (1998, hereafter VM98) who used GHRS echelle data. VM98 reported a value of $\mathrm{D} / \mathrm{H}=0.9 \times 10^{-5}$ for two non-LIC interstellar components observed toward the white dwarf G191-B2B ( $d=69 \mathrm{pc})$, implying $\sim 30 \%$ local variation in the $\mathrm{D} / \mathrm{H}$ ratio. Variations of the $\mathrm{D} / \mathrm{H}$ ratio in the LISM would complicate the use of deuterium as a cosmological probe and affect Galactic chemical evolution model calculations.

We re-examined the $\mathrm{D} / \mathrm{H}$ ratio toward G191-B2B using newer HST-Space Telescope Imaging Spectrograph (STIS) data along with the archival HSTGHRS echelle data (Sahu et al. 1999; hereafter S99). The STIS data of other interstellar species indicate the presence of only two velocity components and the $\mathrm{D} / \mathrm{H}$ ratios derived for the two velocity components are consistent with previous determinations (L93 \& L95). For the second (non-LIC) component, the STIS data yield $\mathrm{D} / \mathrm{H}>1.26 \times 10^{-5}$, which although poorly constrained, is consistent with the LIC value. The STIS echelle data provide no evidence for local or component-to-component variation of the $\mathrm{D} / \mathrm{H}$ ratio in the LISM. We found a clear disagreement between the $\mathrm{D} / \mathrm{H}$ ratios derived from GHRS and STIS data.

In this paper, we present some of the results from S99 together with a brief report on recent progress in the G191-B2B D/H study.

\section{Overview of the G191 B2B Sightline}

The Sun is embedded within the Local Interstellar Cloud (LIC), a warm ( $\mathrm{T}$ $7,000 \mathrm{~K})$, low-density $\left(\mathrm{n}_{e} \sim 0.1\right)$, partially ionized region, which is observed in projection toward most, but not all, nearby stars (Figure 1). Models of the LIC (Redfield \& Linsky, 2000) show the Sun is located just inside the edge of the LIC in the direction of the Galactic Center and toward the North Galactic Pole (NGP). The Hipparcos based distance to G191-B2B is 69 pc (Vauclair et al, 1997). The position of $\mathrm{G} 191-\mathrm{B} 2 \mathrm{~B}\left[(l, b)=\left(155^{\circ} .9,+7^{\circ} .1\right)\right]$ is relatively close to Capella $\left[(l, b)=\left(162^{\circ} .6,+4^{\circ} .6\right)\right]$ in the sky although Capella at $d=12.9 \mathrm{pc}$, is much closer to the Sun. The results obtained for Capella [L93, L95] are relevant for interpreting the G191-B2B data. The $19.5 \mathrm{~km} \mathrm{~s}^{-1}$ component present in the G191-B2B data ( $\S 3.3)$ is at the projected velocity of the LIC and also seen in the Capella data (L93), suggesting that both the G191-B2B and Capella sightlines intercept the LIC.

\section{Our Approach}

Our analysis differs from the VM98 analysis in three main aspects which are listed below.

\subsection{The Radial Velocity of G191-B2B}

The radial velocity of G191-B2B that we use in our analysis is estimated from STIS data of other WD photospheric lines (Bruhweiler et al. 2000, in prepara- 


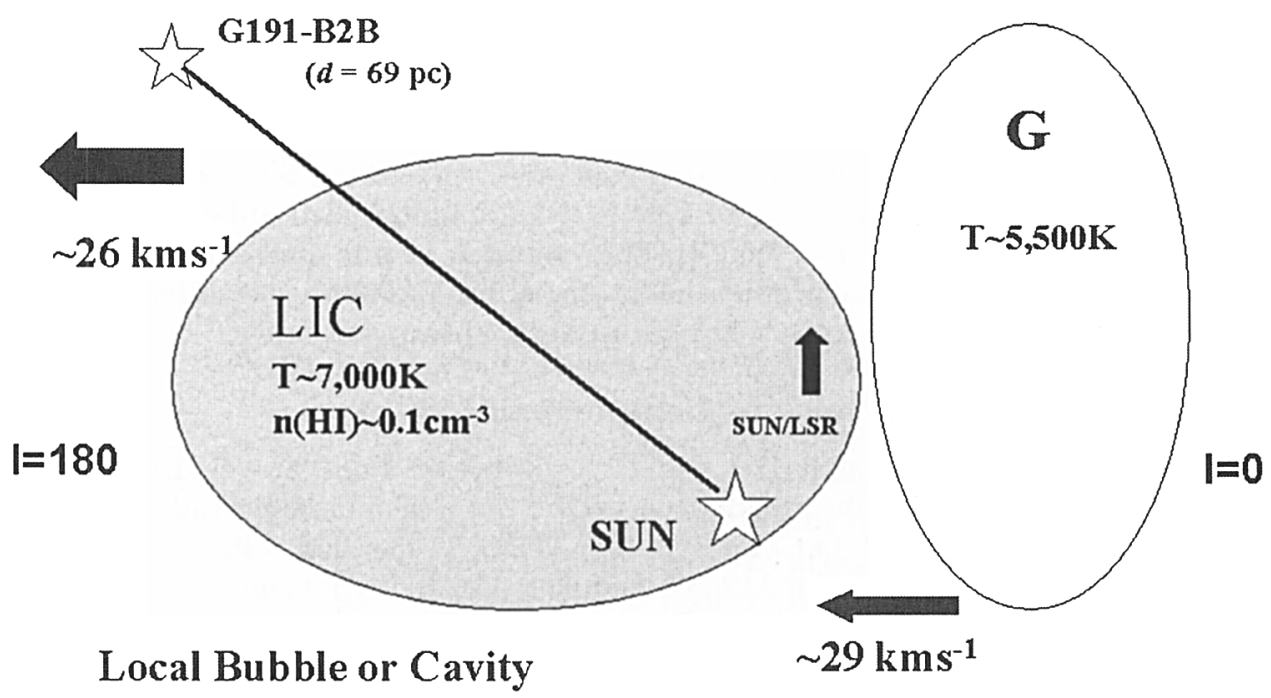

$\mathrm{T} \sim 100$ to $10^{6} \mathrm{~K}$

$\mathrm{n}(\mathrm{HI}) \sim 0.002$ to $500 \mathrm{~cm}^{-3}$

Figure 1. A schematic view of the immediate vicinity of our Sun as viewed from above the Galaxy, shows the relative motions of the Local Standard of Rest (LSR), the Sun, LIC and G clouds. The shapes of the $L I C$ and $G$ clouds as shown here are only for the purpose of illustration and do not represent their true shapes.

tion) and is $24.6 \pm 0.4 \mathrm{~km} \mathrm{~s}^{-1}$ (including gravitational redshift). This differs by $\sim 4.4 \mathrm{~km} \mathrm{~s}^{-1}$ from the value of $29 \mathrm{~km} \mathrm{~s}^{-1}$ derived by VM98. In their analysis, the Si III $1206.5 \AA$ stellar feature, which has low $\mathrm{S} / \mathrm{N}(\sim 10)$ in the GHRS data and is blended with the interstellar contribution has been used to get a radial velocity of $29 \mathrm{~km} \mathrm{~s}^{-1}$ (see $\S 3.1$ of VM98). The STIS wavelength coverage for G191-B2B is $\sim 20$ times more than the GHRS coverage and Bruhweiler et al. use several photospheric lines (mainly $\mathrm{C}, \mathrm{N}, 0, \mathrm{Si}, \mathrm{Fe}$ and $\mathrm{Ni}$ ) in the $\sim 1150-1700 \AA$ range. They obtain a peak for the velocity distribution of $24.6 \mathrm{~km} \mathrm{~s}^{-1}$ with a FWHM of $1.3 \mathrm{~km} \mathrm{~s}^{-1}$. This value is consistent with the radial velocity of $22 \pm 2 \mathrm{~km} \mathrm{~s}^{-1}$ obtained for G191-B2B by Reid \& Wegner (1988) from the H-alpha emission core. Furthermore, Holberg et al. (1998) report an average radial velocity of $21.98 \pm 0.54 \mathrm{~km} \mathrm{~s}^{-1}$ obtained from high-dispersion IUE spectra of metal lines. This average value and the range of velocities quoted in their Table 4 from the different metal lines are consistent with the Bruhweiler et al. (2000) values.

\subsection{Use of NLTE Stellar Atmosphere Models}

Using a physically realistic model to predict the intrinsic stellar Lyman- $\alpha$ profile for G191-B2B is crucial for the $\mathrm{D} / \mathrm{H}$ ratio measurement because the model calculations provide the continuum against which the interstellar D I and H II absorptions are measured. In their analysis VM98 used a pure hydrogen LTE model with $\mathrm{T}_{\text {eff }} \sim 61,400 \mathrm{~K}$ and $\log g \sim 7.55$ and no photospheric metals. The 
GHRS data used by VM98 to determine the Lyman- $\alpha$ photospheric line shape covers only a few $\AA$ of data which do not extend out to the continuum. In contrast, in our analysis we have used stratified, line-blanketed NLTE model calculations by Barstow, Hubeny and co-workers fitted to the STIS data covering the 1140 to $1700 \AA$ region. The best-fit NLTE model atmosphere [ $\mathrm{T}_{e f f}=$ $54,000 \pm 2000 \mathrm{~K}$ and $\log g=7.5 \pm 0.03]$ is adopted in our analysis to predict the intrinsic WD Lyman- $\alpha$ profile and to check for possible contamination of the interstellar lines by narrow WD photospheric absorption lines.

\subsection{The Number of Velocity Components}

In addition to the interstellar D I and $\mathrm{H}$ I absorption lines, the STIS echelle spectra show interstellar absorption due to N I $(\lambda \lambda 1199.5,1200.2$ and 1200.7), C II $\lambda 1334.5, \mathrm{C}^{*}$ II $\lambda 1335.7, \mathrm{O}$ I $\lambda 1302, \mathrm{Si}$ II $(\lambda \lambda 1190,1193,1260,1304$ and 1526), Si III $\lambda 1206.5, \mathrm{Al}$ II $\lambda 1670.8, \mathrm{~S}$ II $\lambda 1259.5$ and Fe II $\lambda 1608.5$. As mentioned in $\S 3.2$, the stratified NLTE calculations predict weak contaminations in several of the interstellar lines mainly by WD photospheric Fe and $\mathrm{Ni}$ absorption lines. The wavelength regions near the interstellar N I $\lambda 1200.7$, Si II $\lambda \lambda 1193 \& 1304$ and Fe II lines show no contamination by WD photospheric lines. The number and relative velocity spacing of individual components that make up an absorption line profile were estimated by an initial inspection of the STIS spectra.

All velocities quoted in this paper are in the heliocentric frame. One velocity component is at $\sim 8.6 \mathrm{~km} \mathrm{~s}^{-1}$ (hereafter referred to as comp 1) and the other is at $19.5 \mathrm{~km} \mathrm{~s}^{-1}$, the projected velocity of the LIC (Lallement et al. 1995) in the line-of-sight to G191-B2B (see $\S 2$ ). Our analysis of the STIS and GHRS data explicitly assumes the existence of two distinct absorption components.

\section{Profile fitting of the interstellar $\mathrm{D}$ I and $\mathrm{H}$ I lines}

Each velocity component is assumed to be homogeneous and characterized by a column density $N$, radial velocity $v$ and a line-of-sight velocity dispersion defined by $b=\left(2 \mathrm{kT} / m+\xi^{2}\right)^{1 / 2}$ where $\xi$ is the turbulent velocity parameter along the line-of-sight, $\mathrm{T}$ is the kinetic temperature and $m$ is the ion mass. In our analysis, we have included the fact that the D I and H I lines are doublets and the two components of each doublet are separated by $0.0054 \AA$. We have simultaneously fitted both the D I and H I interstellar lines since they are separated by only $0.33 \AA$ and the D I absorption is located on the wing of the broad $\mathrm{H}$ I absorption. For each interstellar species, intrinsic line profiles were computed assuming two absorption components and then convolved with either the STIS instrumental LSF for the $0.2 \times 0.2$ arcsec slit given by the STIS Handbook (Sahu, 1999) or the two-component Gaussian LSF for GHRS given by Spitzer \& Fitzpatrick (1993). Three parameters $(N, v, b)$ were determined for each component by an iterative least-squares fit. The turbulent velocity parameters for the two components were determined by plotting the velocity dispersions ( $b$ values) for the various atomic species (D, N, O, C, Si, S, Al and Fe) as a function of ion mass $m$ and performing a least-squares fit to the data points. The best-fit value of $\xi$ for the LIC component is $1.7 \mathrm{~km} \mathrm{~s}^{-1}$ (consistent with the value derived for this component by L93, L95) while for comp $1, \xi$ is $2.5 \mathrm{~km} \mathrm{~s}^{-1}$. 
For modeling of the Lyman- $\alpha$ profile, the velocities of the two components are kept fixed at 8.6 (comp 1) and $19.5 \mathrm{~km} \mathrm{~s}^{-1}$ (LIC) and the $\xi$ values of two components are fixed at 1.7 (LIC) and $2.5 \mathrm{~km} \mathrm{~s}^{-1}$ (comp 1). The results of the profile fitting analysis are listed in S99.

\section{Some new results}

Subsequent to the S99 analysis, several refinements have been made to the IDLbased CALSTIS data reduction package developed by the STIS Instrument Development Team (IDT) which is used to process the STIS data. These refinements include updated dispersion coefficients and improved on-board Doppler corrections. The use of these updated dispersion coefficients has resulted in velocity shifts for the various interstellar absorption lines of up to $\pm 0.3 \mathrm{~km}$ $\mathrm{s}^{-1}$ for G191-B2B as compared to the results published in S99. The velocity shifts introduced due to improved on-board Doppler corrections for the G191B2B data is insignificant, $0.006 \mathrm{~km} \mathrm{~s}^{-1}$ and $0.002 \mathrm{~km} \mathrm{~s}^{-1}$ for the $\lambda_{c}=1234$ and $1598 \AA$ settings respectively and $0.2 \mathrm{~km} \mathrm{~s}^{-1}$ for the $1426 \AA$ setting. The version of IDT-CALSTIS used here is as of 17 December 1999. The derived $\mathrm{D} / \mathrm{H}$ ratios parameters using the new reduction are not significantly different from the values published in S99 and do not affect our earlier conclusions.

In addition to this new analysis, we have also re-analyzed the STIS data using the alternate scattering algorithm provide by Howk \& Sembach (2000). The $\mathrm{D} / \mathrm{H}$ ratios derived using the Howk \& Sembach algorithm is consistent with the $\mathrm{D} / \mathrm{H}$ ratio derived using the IDT reduction. The STIS derived $\mathrm{D} / \mathrm{H}$ ratios $d o$ not agree with the GHRS-derived values irrespective of the scattering algorithm used (Sahu et al. 2000, in preparation).

Acknowledgments. L. da Silva, M. Spite and the Scientific and Local Organizing committees deserve great credit for their organization of this successful meeting.

\section{References}

Burles, S., \& Tytler, D. 1998, ApJ, 499, 699

Dring, A.R. et al. 1997, ApJ, 488, 760

Epstein, R. Lattimer, J., \& Schramm, D. N. 1976, Nature, 263, 198

Holberg, J.B., Barstow, M.A. \& Sion E.M. 1998, ApJS, 119, 207

Howk,C.J. \& Sembach, K.R. 2000, AJ, 119, 248

Lallement, R. et al. 1995, A\&A, 286, 898

Landsman, W. et al. 1996, Science with the Hubble Space Telescope-II, 454

Linsky, J. L. 1998, Space Sci.Rev., 84, 285

Linsky, J.L. et al. 1993, ApJ, 402, 694

Linsky, J.L. et al. 1995, ApJ, 451, 335

Redfield, S. \& Linsky, J.S. 1999, ApJ(in press)

Reid, N. \& Wegner, G. 1988, ApJ, 335, 953 
Reeves, H. et al. 1973, ApJ179, 909

Sahu, M.S. et al. 1999, ApJ, 523, L159 (S99)

Spitzer, L. \& Fitzpatrick,E.L. 1993, ApJ, 409, 299

Vauclair, S. et al. 1997, A\&A325, 1055

Vidal-Madjar, A. et al. 1998, ApJ, 338, 694 (VM98) 